\title{
Large-Scale Anisotropy of TeV-band Cosmic Rays
}

\author{
Rahul Kumar* \\ Physics Department, Ben-Gurion University, Be'er-Sheba 84105, Israel \\ E-mail: rahuliitk@gmail.com
}

\begin{abstract}
The expected anisotropy in the 1 to $10^{4} \mathrm{TeV}$ energy range is calculated for Galactic cosmic rays with anisotropy in the diffusion tensor and source discreteness both taken into account. We find that if the sources are distributed radially (but with azimuthal symmetry) in proportion to Galactic pulsars, the expected anisotropy almost always exceeds the observational limits by one order of magnitude in the case of isotropic diffusion. If the radial diffusion is more than an order of magnitude smaller than the azimuthal diffusion rate, the radial gradient of the sources can be accommodated about 5 percent of the time. If the sources are concentrated in the spiral arms then the anisotropy depends on our location between them, but in some spatial window, roughly equidistant from adjacent spiral arms, the observational constraints on anisotropy are obeyed roughly 20 to 30 percent of the time for extremely anisotropic diffusion. The Solar System is in that window less than 10 percent of the time, but may be there now.
\end{abstract}

The 34th International Cosmic Ray Conference,

30 July- 6 August, 2015

The Hague, The Netherlands

${ }^{*}$ Speaker. 


\section{Introduction}

It is widely agreed that the cosmic rays (CR) of energy below $10^{14.5}$, the so-called knee, are accelerated through diffusive shock acceleration process in supernova remnants (SNRs) in the Galaxy. Recent observations of characteristic pion decay in molecular clouds seem to strengthen the case for Galactic SNRs [1] below $100 \mathrm{GeV}$, but the inferred proton spectra seems to steepen at higher energy. In any case, it remains unproven that supernovae contribute all or even most of the Galactic CRs up to the knee.

Here we present as model considering anisotropic propagation of CRs in the Galaxy together with source discreteness [2]. We find a time dependent analytical estimate of CR density in the Galaxy due to a point-like source. In order to take the discreteness of Galactic CR sources into account, we calculate the net anisotropy of CRs at our location due to randomly placed point-like sources in the Galaxy with a given source distribution and source rate. We compare the anisotropy in case of an isotropic diffusion with the case of anisotropic diffusion with a relatively small radial diffusion rate. It is shown that the systematic anisotropy is greatly reduced in the latter case, while the discreteness anisotropy rises somewhat.

\subsection{Anisotropic Diffusion in Disk Geometry}

We assume a toroidal GMF and adopt a Galacto-centric polar coordinates $\rho, \phi$, and z (z-axis is perpendicular to the Galactic plane and the origin is at the Galactic center) for the following discussions. The differential density $\mathrm{N}$ of $\mathrm{CRs}$ that are instantly released from a source located at $(\rho, \phi, z) \equiv\left(\rho_{0}, 0, z_{s}\right)$ obeys the continuity equation which can be written in the Galacto-centric cylindrical polar coordinate as,

$$
\begin{aligned}
\frac{\partial N}{\partial t}= & \frac{\partial}{\rho \partial \rho} \rho D_{\rho} \frac{\partial N}{\partial \rho}+\frac{\partial}{\rho^{2} \partial \phi} D_{\phi} \frac{\partial N}{\partial \phi}+\frac{\partial}{\partial z} D_{z} \frac{\partial N}{\partial z} \\
& +Q(E) \delta(t) \delta\left(\rho-\rho_{0}\right) \delta(\phi) \delta\left(z-z_{s}\right) / \rho
\end{aligned}
$$

where $\mathrm{Q}(\mathrm{E})$ is the differential production rate of $\mathrm{CRs}$, and $D_{\rho}, D_{\phi}$, and $D_{z}$ are the radial, azimuthal, and axial diffusion rates, respectively. CRs reaching a few Kpc out into the halo efficiently escape out of the Galaxy because of a weak local GMF which is insufficient to scatter CRs. Therefore, we introduce absorbing boundary conditions $N(z \pm H)=0$ to encapsulate escape of CRs out of the face of the Galaxy. To find the Green's function for the diffusion equation 1.1, we separate the differential density $N(\rho, \phi, z, t)$ into mid plane density $N_{0}(\rho, \phi, t)$ and $N_{z}(z, t)$, i.e

$$
N(\rho, \phi, z)=N_{z}(z, t) N_{0}(\rho, \phi, t) .
$$

Using the standard method of mirror images to satisfy the absorbing boundary condition at $z= \pm H$, $N_{z}(z, t)$ can be written as,

$$
N_{z}=\frac{(-1)^{n}}{\sqrt{4 \pi D t}} \exp \left(-\frac{\left(z-2 n H-(-1)^{n} z_{s}\right)^{2}}{4 D t}\right),
$$

which, as described in [3], can then be approximated (for $z_{s}<<H$ ) with good accuracy as 


$$
N_{z} \simeq \frac{1}{\sqrt{4 \pi D t}} \exp \left(-\frac{\left(z-z_{s}\right)^{2}}{4 D t}\right)(1+\tilde{t})^{1.25} \exp \left(-(1.5 \tilde{t})^{0.97},\right.
$$

where $\tilde{t}=2 D t / H^{2}$. We find a series solution to the two dimensional diffusion equation for the mid-plane density $N_{0}$, which can be written as

$$
\begin{aligned}
N_{0}(\rho, \phi, t)= & \frac{\Theta(t) Q(E)}{2 \pi D_{\rho} t} \exp \left(-\frac{\rho^{2}+\rho_{0}^{2}}{4 D_{\rho} t}\right) \times \\
& \times\left[\frac{1}{2} I_{0}(\tilde{\rho})+\sum_{n=1}^{\infty} \cos (n \phi) I_{v(n)}(\tilde{\rho})\right],
\end{aligned}
$$

where $\tilde{\rho}=\rho \rho_{0} / 2 D_{\rho} t$, and $v(n)=n \sqrt{D_{\phi} / D_{\rho}}$. For a given diffusion parameters, which depends on the CR energy, equations $1.2,1.4$, and 1.5 combined is used to calculate CR flux at the Earth due to a point-like source in the Galaxy.

\section{Cosmic Ray Flux Anisotropy}

In figure 1, for a comparison between anisotropic and isotropic diffusion, we have plotted all three orthogonal components of the dipole anisotropy at $20 \mathrm{TeV}$ in both cases, assuming a steep source distribution $S_{T}$. It is clear from the top panel, which is for isotropic diffusion, the radial anisotropy is the dominant contributor (about 10 times larger than the azimuthal anisotropy) to the total anisotropy. As the radial diffusion rate is reduced, the systematic anisotropy due to large scale inhomogeneity in source distribution reduces in proportion. Discreteness anisotropy due to source discreteness however remains. As is evident from the bottom panel in figure 1, the radial anisotropy comes down to the level of azimuthal anisotropy if the radial diffusion rate is reduced by a factor of 10. As the radial diffusion rate is reduced the radial distance to which CRs can reach before escaping out of the face of the Galaxy is also reduced, which implies a relatively smaller number of sources contributing to the total flux at any given instant. A smaller number of contributing sources leads to a modest increase in the discreteness anisotropy, which slightly offsets the decrease in total anisotropy due to a reduced radial diffusion rate.

\section{References}

[1] Ackermann, M. et al., 2013, Science, 339, 6121

[2] Kumar R. and Eichler D., 2014, The Astrophysical Journal, 785,129

[3] Pohl M. and Eichler D. 2013, The Astrophysical Journal, 766, 1 


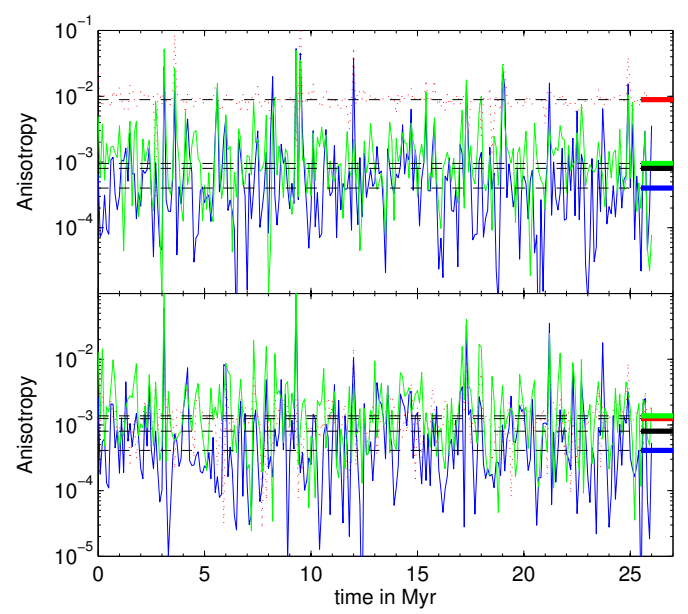

Figure 1: Temporal variation of all three orthogonal components of anisotropy, namely, radial (red), azimuthal (green), and axial component (blue) is plotted for a) top panel: isotropic diffusion, and b) bottom panel: anisotropic diffusion with $D_{\rho}=D^{i s o} / 10$. The thick colored bars at the right of each panels, extended as dashed black lines, indicate median anisotropy of each component of the anisotropy with corresponding color. Here, and henceforth, the source rate is 1 per 100 years, the source distribution is assumed to be $S_{T}$, and half disk thickness $\mathrm{H}$ is $5 \mathrm{kpc}$.

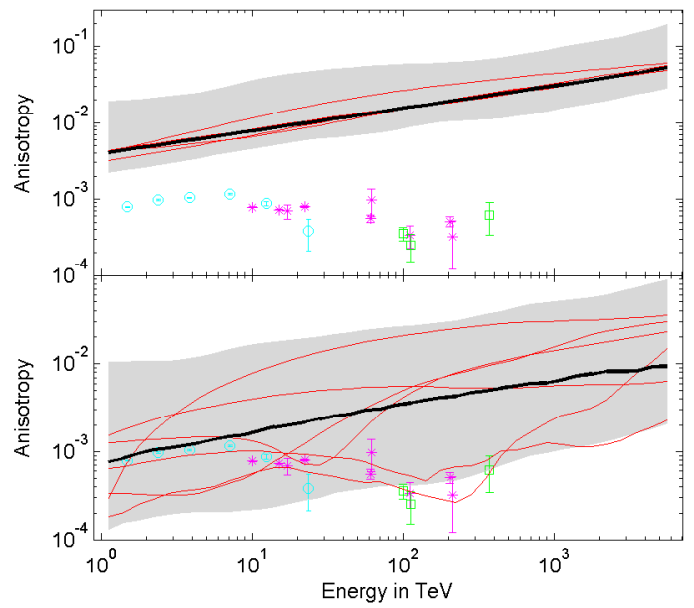

Figure 2: Total dipole anisotropy is plotted for a) top panel: isotropic diffusion, and b) bottom panel: anisotropic diffusion with $D_{\rho}=D^{i s o} / 50$. The red curves show the variation of anisotropy with energy for randomly selected realisations of source distribution. The thick black line indicates median value of the anisotropy at any given energy over a large ensemble of source distributions and the shaded area indicates 95\% fluctuation region. Measurements from ARGO-YBJ (cyan), TIBET III (purple), and muon shower experiments (green) are shown. 